University of Wollongong

Research Online

Faculty of Engineering and Information

Faculty of Engineering and Information

Sciences - Papers: Part B

Sciences

2018

\title{
Effect of Rubber Crumbs on the Cyclic Behavior of Steel Furnace Slag and Coal Wash Mixtures
}

\author{
Yujie Qi \\ University of Wollongong, qyujie@uow.edu.au \\ Buddhima Indraratna \\ University of Wollongong, indra@uow.edu.au \\ Ana Heitor \\ University of Wollongong, aheitor@uow.edu.au \\ J S. Vinod \\ University of Wollongong, vinod@uow.edu.au
}

Follow this and additional works at: https://ro.uow.edu.au/eispapers1

Part of the Engineering Commons, and the Science and Technology Studies Commons

Research Online is the open access institutional repository for the University of Wollongong. For further information contact the UOW Library: research-pubs@uow.edu.au 


\title{
Effect of Rubber Crumbs on the Cyclic Behavior of Steel Furnace Slag and Coal Wash Mixtures
}

\author{
Abstract \\ The practical application of waste materials such as steel furnace slag (SFS) and coal wash (CW) is \\ becoming more prevalent in many geotechnical projects. While adding rubber crumbs (RCs) from \\ recycled tires into mixtures of SFS and CW not only solves the problem of large stockpiles of waste tires, \\ it also can provide an energy-absorbing medium that will reduce vibration and prevent track degradation. \\ Thus, the engineering insight into the effect that rubber crumbs have on the dynamic behavior of SFS $p$ \\ $\mathrm{CW}$ p RC mixtures is in urgent demand. In this study the influence that RC contents and confining \\ pressures have on the deformation, resilient modulus, damping ratio, and shear modulus was investigated \\ by cyclic triaxial tests. Test results reveal that with the inclusion of RC, the axial strain, volumetric strain, \\ damping ratio, and energy-absorbing capacity of the SFS p CW p RC mixture increase, while its resilient \\ modulus and shear modulus decrease. Based on these properties, an amount of $10 \%$ RC is recommended \\ as an optimal blended mix to be used as railway subballast. A three-dimensional (3D) empirical model of \\ the relationship between the maximum axial strain, volumetric strain, and resilient modulus with RC \\ contents and the effective confining pressure was developed, and the energy-absorbing capacity of these \\ waste mixtures has also been analyzed for practical purposes based on their comprehensive parameters.

\section{Disciplines} \\ Engineering | Science and Technology Studies

\section{Publication Details} \\ Qi, Y., Indraratna, B., Heitor, A. \& Vinod, J. S. (2018). Effect of Rubber Crumbs on the Cyclic Behavior of \\ Steel Furnace Slag and Coal Wash Mixtures. Journal of Geotechnical and Geoenvironmental Engineering, \\ 144 (2), 04017107-1-04017107-11.
}




\title{
The Role of Rubber Crumbs on the Cyclic Behavior of Steel Furnace Slag and Coal Wash Mixtures
}

\author{
Yujie Qi \\ PhD Candidate
}

Centre for Geomechanics and Railway Engineering, Faculty of Engineering and Information

Sciences, University of Wollongong, Wollongong, NSW 2522, Australia

\section{Buddhima Indraratna}

PhD (Alberta), MSc and BSc-Hons (London), FTSE, FIEAust, FASCE, FGS, FAusIMM, FIES, DIC, CEng, CPEng

Distinguished Professor, Research Director and Foundation Director, Civil Engineering, Centre for Geomechanics and Railway Engineering, Faculty of Engineering and Information Sciences, University of Wollongong, Wollongong, NSW 2522, Australia

\section{Ana Heitor}

$\mathrm{PhD}$

Senior Lecturer, Centre for Geomechanics and Railway Engineering, Faculty of Engineering and Information Sciences, University of Wollongong, Wollongong, NSW 2522, Australia

\section{Jayan S. Vinod}

$\mathrm{PhD}$

Senior Lecturer, Centre for Geomechanics and Railway Engineering, Faculty of Engineering and Information Sciences, University of Wollongong, Wollongong, NSW 2522, Australia

\author{
$\dagger$ Author for correspondence: \\ Prof. Buddhima Indraratna \\ School of Civil Engineering, \\ Faculty of Engineering, University of Wollongong \\ Wollongong, NSW 2522 \\ AUSTRALIA \\ Ph: +61242213046 \\ Fax: +612 42213238 \\ Email: indra@uow.edu.au
}

To be submitted to: Journal of Geotechnical and Geoenvironmental Engineering 
1 Abstract: The practical application of waste materials such as steel furnace slag (SFS) and

2 coal wash $(\mathrm{CW})$ is becoming more prevalent in many geotechnical projects. While adding

3 rubber crumbs (RC) from recycled tyres into mixtures of SFS and CW not only solves the

4 problem of large stockpiles of waste tires, it also can provide an energy absorbing media that

5 will reduce vibration and prevent track degradation. Thus the engineering insight into the

6 effect that rubber crumbs has on the dynamic behavior of SFS+CW+RC mixtures is in urgent

7 demand. In this study the influence that RC contents and confining pressures have on the

8 deformation, resilient modulus, damping ratio, and shear modulus was investigated by cyclic

9 triaxial tests. Test results reveal that with the inclusion of $\mathrm{RC}$ the axial strain, volumetric

10 strain, damping ratio and the energy absorbing capacity of the SFS+CW+RC mixture

11 increase, while its resilient modulus and shear modulus decrease. Based on these properties,

12 an amount of $10 \% \mathrm{RC}$ is recommended as an optimal blended mix to be used as railway

13 subballast. A 3D empirical model of the relationship between the maximum axial strain,

14 volumetric strain, and resilient modulus with $\mathrm{RC}$ contents and the effective confining

15 pressure was developed, and the energy absorbing capacity of these waste mixtures has also

16 been analysed for practical purposes, based on their comprehensive parameters.

17 KEYWORDS: Steel furnace slag; coal wash; rubber crumbs; cyclic loading; resilient

18 modulus; damping ratio; shear modulus; energy absorbing capacity 
Steel furnace slag (SFS) and coal wash (CW) are waste materials that derive from steel making and coal mining industries. In the Wollongong region (Australia) alone, the production of granular waste materials SFS and CW amount to more than $2 \mathrm{mt}$ per year (Chiaro et al., 2015). One of the best ways to deal with the large amounts of SFS and CW wastes is to reuse them as construction materials in geotechnical projects. However, the individual detrimental characteristics of these materials, i.e. swelling potential of SFS and the particle breakage of CW prevent them from being used on its own.

To optimise the geotechnical properties, SFS and CW are usually blended with other materials prior to their adoption as a structural fill. For instance, $10 \%$ Class $\mathrm{C}$ fly ash has been added to steel slag by Yildirim \& Prezzi (2015) to serve as landfill. However, Juan et al. (2011) have mixed steel slag with Portland cement, and Malasavage et al. (2012) have blended steel slag fines with dredged materials. The steel slag can also be mixed with asphalt or concrete for practical applications in unbound pavements (Xue et al., 2006). Moreover, mixtures of SFS and CW prepared at optimal blending ratios have been reported to overcome their inherent weaknesses, and the SFS+CW mixtures have been successfully used in practical engineering applications such as port reclamation (Chiaro et al., 2015; Tasalloti et al., 2015) and landfill projects (Indraratna et al., 1994, Heitor et al., 2016). Despite these successes, previous studies on the use of SFS and CW have been limited to monotonic loading conditions.

Another issue concerns the geoenvironmental pollution caused by SFS and CW. Based on trace element concentration tests, neither coal wash nor steel furnace slag poses any significant risk of environmental contamination. The commercial use of these engineered fills above and below the groundwater level has already been approved by the Environment 
43 Protection Authority of the state of New South Wales (NSW EPA, 2014). Similarly, chemical test results reported by Lim and Chu (2006) indicate that the heavy metal concentrations contained in a typical steel slag leachate were significant lower than the threshold toxicity limits stipulated by the US EPA.

Waste tyres stockpiles can lead to serious environmental problems, especially in developing countries. The pressing need therefore, from an environmental perspective, is to encourage the re-use of this waste material in innovative ways. Rubber crumbs (RC) or granulated rubber from waste tyres has been found to have a low unit weight, high hydraulic conductibility, high elastic deformability, low shear strength, and high energy absorbing capacity (Senetakis et al., 2012; Zheng \& Kevin, 2000). The relatively high damping property of rubber materials makes the tyre rubber-soil mixture a promising material for reducing vibration and noise. Tsang et al. (2012) conducted numerical investigations to examine the potential of a tyre rubber-soil mixture for seismic conditions. They found this mixture was able to reduce the vibration efficiently by $40-60 \%$. Other studies have also shown that tyre rubber-soil mixtures can be considered as an integral part of vibration damping systems for machine foundations and railroads (Lee, et al., 1999; Li et al., 2016; Zheng \& Kevin, 2000; Sheikh et al., 2013).

The inclusion of RC into SFS and CW extends the application of these waste mixtures into dynamic loading conditions to reduce vibration, prevent track degradation, and absorb energy, all due to the high damping ability of rubber. This application means the effect that rubber has on the dynamic behavior (especially during cyclic loading) of SFS+CW+RC mixtures must be investigated in depth. Although a number of researches have already examined the dynamic behavior of rubber-soil mixtures, most of them only focus on small strain behavior $(\leq 0.1 \%$ shear strain) by resonant column tests or shaking table tests (e.g. Senetakis et al., 2012; Zheng \& Kevin, 2000; Leo \& Mladen, 2003). Such small strains are not directly 
applicable to rapidly densifying granular assemblies under cyclic loading (shear strain > 0.1\%). Li et al. (2016) studied the dynamic behavior of rubber-sand mixtures under cyclic loading, but the level of deformation achieved under a large number of loading cycles, relevant to transport infrastructure applications, was not considered. Moreover, the study of the energy absorbing capacity of these mixtures due to the inclusion of RC needs to be examined in detail based on comprehensive test results.

This paper aims to investigate the influence of rubber crumbs on the deformation behavior, resilient modulus, damping ratio, and shear modulus under cyclic loading of the SFS, CW mixtures. Based on the cyclic loading properties of the blended waste mixtures, the potential use of SFS+CW+RC mixtures as an alternative subballast material to replace traditional capping materials (i.e. compacted gravels and coarse sands) will be explored. In addition, the energy absorbing capacity of waste mixtures having different RC contents will be studied comprehensively.

\section{Laboratory Investigations}

\section{Materials}

The source materials consist of Dendrobium coal wash from Illawarra Coal and an SFS from ASMS (Australia Steel Milling Services), respectively. The rubber crumbs were obtained through shredding of waste tyres and in this study three different sizes $(0-2.3 \mathrm{~mm}, 0.3-3 \mathrm{~mm}$, and 1-7 mm) were used. Coal wash is predominantly composed quartz and residual coal, with illite and kaolinite as the main clay minerals. Trace quantities of calcite, pyrites and sulphur were also detected in the x-ray diffraction analysis. The chemical composition is given in Table 1. The CW aggregates compose of both angular and relatively flaky grains, and typically exhibits dual porosity. The steel furnace slag is composed mainly of metal 
91 compounds (e.g. $\left.\mathrm{Fe}_{2} \mathrm{O}_{3}, \mathrm{SiO}_{2}\right)$ and free lime $(\mathrm{CaO})$, and the chemical composition determined by X-ray diffraction analysis is also shown in Table 2.

The particle size distribution (PSD) curves of SFS, CW, and RC are shown in Fig. 1. The dry method was used to sieve the oven-dried SFS and air-dried rubber crumbs whereas the wet method was used for CW. SFS and CW can be classified as well-graded gravel with siltysand (GW-GM), and well-graded sand with gravel (SW) (unified soil classification system, USCS), respectively; while the RC can be referred to as granulated rubber (ASTM D6270, 2008). The inclusion of rubber crumbs in this study is limited to $40 \%$ by weight (which corresponds to about $60 \%$ by volume), because a rubber content of more than $60 \%$ by volume means the mixtures generally exhibit a rubber-like behavior as the rubber-to-rubber interfaces develop, and the overall dynamic behavior is mainly controlled by the rubber (Senetakis et al., 2012; Kim et al., 2008). In these cases the mixtures undergo high compressibility and exhibit a low shear strength which is not recommended for transport applications. Moreover, the ratio of SFS:CW in this paper is set at $7: 3$ because there is less particle breakage, less volumetric expansion, and enough shear strength.

The basic geotechnical properties (specific gravity $G_{s}$, maximum dry density $\gamma_{\mathrm{dmax}}$, optimum moisture content OMC, and California Bearing Ratio CBR) of SFS, CW, RC, and their mixtures are shown in Table 3. The basic geotechnical properties of the waste mixtures indicate that SFS is the heaviest material, while RC is the lightest among the three waste materials. Therefore, as $\mathrm{RC}$ content increases in SFS+CW+RC mixtures, the specific gravity $G_{s}$ as well as the maximum dry density $\gamma_{d \max }$ of the waste mixture decrease. The optimum moisture content $\mathrm{OMC}$ is $11.5-15 \%$ for $\mathrm{SFS}+\mathrm{CW}+\mathrm{RC}$ mixtures with $\mathrm{SFS}: \mathrm{CW}=7: 3$ and 0 $40 \%$ RC. The soaked CBR value decreases as more RC is added indicating the high compressibility of rubber materials. 


\section{Specimen preparation and test procedure}

116 To exclude the influence of gradation, all the mixtures tested in this study were mixed to the

117 same gradation (the target PSD), also shown in Fig. 1. Following a previous study by 118 Tasalloti et al. (2015), to achieve the target particle size distribution (PSD), the waste materials were sieved and separated into different particle sizes, and the exact mass corresponding to a given size range was weighed and mixed thoroughly to obtain a uniform blend. All the specimens were prepared at the optimum moisture content and compacted to achieve an initial dry unit weight equivalent to $95 \%$ of their $\gamma_{\mathrm{dmax}}$ (Table 3 ) to simulate typical field conditions of subballast.

A series of stress-controlled drained cyclic triaxial tests were carried out for the SFS+CW+RC mixtures with SFS:CW=7:3 and different amounts of RC $(0 \%, 10 \%, 20 \%$, $30 \%$, and 40\%) following the procedure suggested by ASTM D5311/D5311M (2013). The specimens were compacted in three layers and had $50 \mathrm{~mm}$ in diameter by $100 \mathrm{~mm}$ high. The maximum particle size of the materials is $6.7 \mathrm{~mm}$, thus the ratio of specimen diameter (50 $\mathrm{mm}$ ) to the maximum particle size is around 7.5. Previous studies have shown that the equipment boundary size effects can be neglected when this ratio exceeds at least 6 (Marachi et al., 1972; Indraratna et al., 1994). In this study, an appropriate range of effective confining pressure (i.e. $\sigma_{3}^{\prime}=10,40$, and $70 \mathrm{kPa}$ ) was used to simulate the field conditions of railway subballast depending on the typical axle loads (heavy haul) and heights of track embankments in the state of NSW (Indraratna et al., 2007; Indraratna et al., 2011).

Cyclic loading tests were conducted following three stages, i.e. saturation, consolidation, and cyclic loading. During the saturation stage, the specimens were flooded with de-aired water and then the back pressure was applied at an increasing rate of $1 \mathrm{kPa} /$ minute until $500 \mathrm{kPa}$ was reached. This stage was completed when the Skempton's B-value exceeded 0.98, and 
then isotropic consolidation was carried out until the desired mean effective confining pressure of 10, 40 or $70 \mathrm{kPa}$ was reached. After consolidation, the cyclic loading stage was conducted at $\mathrm{CSR}=0.8$ (cyclic stress ratio, Eq. 1), using a loading frequency of $f=5 \mathrm{~Hz}$. The deviator stress used in this study is governed by $\sigma_{3}^{\prime}$ and cyclic stress ratio, CSR. For $\mathrm{CSR}=0.8$, the confining pressures of ${\sigma^{\prime}}_{3}=10,40$, and $70 \mathrm{kPa}$ correspond to deviator stresses of 16,64 , and $112 \mathrm{kPa}$, respectively. These values are in conformity with the observed stress conditions generated in typical freight tracks (Indraratna et al., 2011).

$$
C S R=\frac{\sigma_{a}}{2 \sigma_{3}^{\prime}}
$$

where, CSR is the cyclic stress ratio; $\sigma_{a}$ is the peak cyclic axial stress; and $\sigma^{\prime}{ }_{3}$ is the effective confining pressure.

The cyclic loading continued for 50000 cycles which is more in line with track inspection and maintenance schedules of real-life tracks in the state of NSW, Australia. Once the tests were completed, the sieving procedure was repeated so that the particle breakage incurred during the cyclic tests could be evaluated.

The resilient modulus $M_{R}$ is determined by the abovementioned permanent deformation cyclic triaxial test. To further eliminate any possible influence of the irregularity in loading happened in the initial stage (Nazzal \& Mohammad, 2010; Lackenby et al., 2007), $M_{R}$ was determined after 1000 cycles where all the specimens reached a constant applied cyclic deviator stress and over $90 \%$ of their permanent vertical strain (Figs. 2a and b). Moreover, the value of $M_{R}$ is determined under saturated condition. This is because in Australia, the magnitude resilient modulus has to meet certain requirements in contractual agreements because of many low-lying coastal tracks in which the subballast is usually saturated by the high groundwater table. Some past studies have also saturated the specimens before 

Indraratna (2015).

\section{Results and Discussion}

\section{Axial strain, volumetric strain, and resilient modulus}

Cyclic loading test results on the permanent deformation and resilient modulus of the $\mathrm{SFS}+\mathrm{CW}+\mathrm{RC}$ mixtures are presented in this section. The axial strain with loading cycles can be used to evaluate the settlement behavior of the waste mixtures under cyclic train moving loads, and the volumetric strain reflects the changes of void ratio. The resilient modulus can be computed based on the load-unload hysteretic cycles.

Figure 2 shows a typical hysteretic response of SFS+CW+RC mixtures having different $\mathrm{RC}$ contents tested at $\sigma_{3}^{\prime}=70 \mathrm{kPa}$. From Fig. 2 (a) and (b), it can be observed that the permanent axial strain increases with the loading cycles but with a decreasing accumulation rate, while the recoverable axial strain decreases with the loading cycles and becomes much more stable after 1000 cycles. The maximum deviator stress $q_{\max , c y c}$ changes with the development of the axial strain at the beginning of each test. With the increasing of loading cycles, the maximum deviator stress becomes much more stable as the axial strain tends to be stable. The hysteretic loops of SFS+CW+RC mixtures having different RC contents at 10000 cycles are shown in Fig. 2 (c), and it is found that the addition of RC significantly influences the deformation of the waste mixtures. As RC contents increase, the area of the hysteretic loops increases, and the inclination also increases indicating the recoverable axial strain increases.

Figure 3 shows the total axial strain $\varepsilon_{1}$ and volumetric strain $\varepsilon_{v}$ which evolves with the logarithm of the number of cyclic loading cycles $\mathrm{N}$. As expected, the axial strain $\varepsilon_{1}$ increases 
with the effective confining pressure $\sigma_{3}^{\prime}$ as $q_{\max , c y c}$ increases (Fig. 3a-c). Further, at the same effective confining pressure, higher $\mathrm{RC}$ contents generate greater total axial strain as both the permanent axial strain and the elastic axial strain increases (e.g. Fig. 2). In the context of rail tracks, shakedown of a granular material is a physical phenomenon where cyclic densification facilitates a relatively stable axial strain after a certain period of time or number of loading cycles (Indraratna et al., 2011). In Fig. 3 (a-c) it can be seen that at lower confining pressures e.g. $\sigma_{3}^{\prime}=10 \mathrm{kPa}\left(q_{\max , c y c}\right.$ is $\left.16 \mathrm{kPa}\right)$, all the waste mixtures tend to achieve relatively stable axial strains or shakedown within about 1000 cycles, and this is also consistent with the past findings reported by Lackenby et al. (2007) for coarser aggregates. At higher confining pressures (i.e. 40 and $70 \mathrm{kPa} ; q_{\max , c y c}$ are 64 and $112 \mathrm{kPa}$, respectively) the waste mixtures with $\mathrm{RC} \leq 10 \%$ shakedown within 10000 cycles, whereas $\varepsilon_{1}$ of those with higher RC contents continues to accumulate although at a marginal rate. The axial strain of all the test specimens reported herein attained a relatively stable state approaching 10000 cycles or beyond until the end of testing (Fig. 3a-c).

The volumetric strain $\varepsilon_{v}$ with the logarithm of cyclic numbers is shown in Fig. 3 (d-e). For each specimen, during the first 10 cycles, the volumetric strain $\left(\varepsilon_{v}=\varepsilon_{1}+2 \varepsilon_{r}\right)$ grows marginally, which is in agreement with findings for other granular materials (Lackenby et al., 2007; Indraratna et al., 2014). In this region, the axial strain $\varepsilon_{1}$ increases (compression) while the radial strain $\varepsilon_{r}$ increases (dilation), therefore, the volumetric strain shows little variation. After 10 cycles, densification starts occurring, $\varepsilon_{1}$ continues to increase and $\varepsilon_{r}$ decreases, thus $\varepsilon_{v}$ undergoes rapid compression except for the mixture having $0 \% \mathrm{RC}$ which begins to dilate around 5000 cycles (Fig. 3d). Within 10000 loading cycles, the behavior of volumetric strain observed is consistent with other granular materials (e.g. Bian et al., 2016; Lackenby et al., 2007), but after $\mathrm{N}=10000$ the volumetric strains seem to be governed mainly by RC contents and the effective confining pressures. 
209 After 10000 cycles, $\varepsilon_{v}$ of waste mixtures with $\mathrm{RC} \leq 10 \%$ stabilises when the specimen is 210 subjected to the higher confining pressure $(40,70 \mathrm{kPa})$ and begins to dilate at lower confining 211 pressure $(10 \mathrm{kPa})$, which is similar to general granular materials (e.g. Bian et al., 2016). For 212 those waste mixtures with $\mathrm{RC}>10 \%, \varepsilon_{v}$ remains increasing at a high rate at $\sigma_{3}^{\prime}=$ 21310 and $40 \mathrm{kPa}$, while at ${\sigma^{\prime}}_{3}=70 \mathrm{kPa} \varepsilon_{v}$ continues to develop but at a declining rate as the 214 number increases. Except the specimen with $0 \% \mathrm{RC}$ tested at $\sigma_{3}^{\prime}=10 \mathrm{kPa}$, all the specimens present a compression behavior, and with the increasing of effective confining 216 pressures and $\mathrm{RC}$ contents, the value of the volumetric strain tend to behave more contractive.

217 It is understood that rubber crumbs have a lower stiffness compared to the mixtures and they show a greater compression with the increase in RC contents (Kim \& Santamarina, 2008; Sheikh et al., 2013). Furthermore, with an increased proportion of RC the elastic deformation within in the rubber crumbs can become increasingly more pronounced at higher load magnitudes (Sheikh et al., 2013; Zheng \& Kevin, 2000). The influence of RC contents on the

222 dilatancy behavior of the waste mix can be also illustrated by plotting the dilatancy $\frac{d \varepsilon_{v}^{p}}{d \varepsilon_{v}^{q}}$ with

223 the stress ratio $\frac{q}{p \prime}$ of SFS+CW+RC mixtures with varying $\mathrm{RC}$ contents (Fig. 4). It clearly 224 shows that increasing the RC content can reduce the dilatancy of the waste mixture.

225 The resilient modulus $M_{R}$ is a very important parameter in depicting the characteristic 226 deformation of materials; it can be defined as

$$
M_{R}=\frac{\Delta q_{c y c}}{\varepsilon_{1, r e c}}
$$

227 where $\Delta q_{c y c}$ is the difference between the maximum cyclic deviator stress $q_{\max , c y c}$ and the 228 minimum cyclic deviator stress $q_{\text {min,cyc }}$, and $\varepsilon_{1, r e c}$ is the recoverable axial strain (elastic 229 axial strain) during unloading. 
230 Figure 5 shows the variation of $M_{R}$ with the number of cycles for the waste mixtures tested at 231 different confining pressures (i.e. 10, 40, and $70 \mathrm{kPa}$ ). $M_{R}$ increases with the number of 232 loading cycles, but then stabilises after a certain number of cycles $(\mathrm{N}=10000)$. This is in 233 agreement with the cyclic behavior of other granular materials (Bian et al., 2016; Lackenby et al., 2007; and Indraratna et al., 2014). As the confining pressure increases, $M_{R}$ increases, possibly because the rubber becomes more compressible under a higher confining pressures where new particle contacts between stiffer particles (SFS and CW) would form (Li et al., 2016). Similarly, Lee et al. (2007) found that at high confining pressures the rubber-sand mixtures tend to behave in a more sand-like manner rather than a rubber-like manner. Furthermore, the $M_{R}$ of the waste mixtures decreases as the amount of RC increases because the inclusion of rubber crumbs creates more rubber-to-rubber contacts, as well as an increase in the recoverable axial strain $\varepsilon_{1, \text { rec }}$ (Senetakis et al., 2012).

242 Excessive deformation will lead to hazardous impact to a ballast track foundation. The average axial strain for traditional railway subballast layer is in the proximity of $2 \%$ (Teixeira et al., 2006). The cyclic loading results (e.g. Figs. 3 and 5) indicate that waste mixtures having $\mathrm{RC} \geq 30 \%$, may be unsuitable for subballast design because the inclusion of additional rubber crumbs leads to an increased permanent deformation and a reduced resilient modulus. Furthermore, these tests also confirm that mixtures of CW and SFS without rubber crumbs cannot be used as subballast due to their significantly high dilation characteristics under relatively low confining pressures $(<40 \mathrm{kPa})$. Moreover, the resilient modulus for subballast is expected to be in the range $60-100 \mathrm{MPa}$ when $q_{c y c}$ is around $80 \mathrm{kPa}$ (Shahu et al., 1999), therefore, a SFS+CW+RC mixture with $10 \%$ RC offers an optimum mixture for a subballast capping layer.

\section{Permanent strain and resilient modulus as a function of $\boldsymbol{R}_{b}(\%)$ and $\sigma^{\prime}{ }_{3}$}


The effect that rubber crumbs $R_{b}(\%)$ has on the cyclic loading behavior of waste mixtures is

255

256

257

258

259

260

261

262

263

264

265

266

267

268

269

270

271 best evaluated by plotting the final values of radial strain $\varepsilon_{r}$, the axial strain $\varepsilon_{1}$, the volumetric strain $\varepsilon_{v}$, and the resilient modulus $M_{R}$ at 10000 cycles (Fig. 6 and Fig. 7) after which the trend of the deformation is stable. The dependence that permanent strains $\left(\varepsilon_{1}\right.$ and $\left.\varepsilon_{v}\right)$ and resilient modulus has on RC contents $R_{b}(\%)$ and the effective confining pressure $\sigma_{3}^{\prime}$ are clearly apparent as described in the previous sections. For the radial strain shown in Fig. 6, it can be seen that $\varepsilon_{r}$ increases as $R_{b}(\%)$ and ${\sigma^{\prime}}_{3}$ increases, and the radial strain at 10 cycles is greater than that at 10000 cycles, which further explain the development of the volumetric strain in Fig. 3(d-f), but the relationship of $\varepsilon_{r}$ with $R_{b}(\%)$ is not as clear. Except the effect that $R_{b}(\%)$ has on $\varepsilon_{r}$ (Fig. 6), the relationship that $R_{b}(\%)$ has on $\varepsilon_{1}, \varepsilon_{v}$, and $M_{R}$ all can be described by certain functions of $R_{b}(\%)$ with a good correlation (Fig. 7). It is noted here that the empirical equations formulated and given in Fig. 7 are only based on the test results of SFS+CW+RC mixtures having $0 \%, 10 \%, 20 \%, 30 \% \mathrm{RC}$, and the test results of waste mixtures having $40 \% \mathrm{RC}$ are considered separately for the purpose of validation.

The relationship that $\varepsilon_{1}, \varepsilon_{v}$, and $M_{R}$ have with RC contents could be presented as (Fig. 7a-c):

$$
\begin{gathered}
\varepsilon_{1}=f_{1}\left(\sigma_{3}^{\prime}\right) *\left(R_{b}\right)^{f_{2}\left(\sigma_{3}^{\prime}\right),} \\
\varepsilon_{v}=f_{3}\left(\sigma_{3}^{\prime}\right) * \ln \left(R_{b}\right)+f_{4}\left(\sigma_{3}^{\prime}\right), \\
M_{R}=f_{5}\left(\sigma_{3}^{\prime}\right) * e^{f_{6}\left(\sigma_{3}^{\prime}\right) * R_{b}}
\end{gathered}
$$

where $f_{1}, f_{2}, f_{3}, f_{4}, f_{5}$ and $f_{6}$ are parameters associated with the effective confining pressure, $\sigma^{\prime}{ }_{3} . R_{b}$ is the content of rubber, $\mathrm{RC}(\%)$. It is interesting to find that the specific parameters $f_{1}$, $f_{2}, f_{3}, f_{4}, f_{5}$ and $f_{6}$ all have a linear relationship with $\sigma_{3}^{\prime}$

$$
f_{n}=\mathrm{k}_{n 1} \cdot \sigma_{3}^{\prime}+\mathrm{k}_{n 2}
$$
in Table 4 . Thus by relating the effective confining pressure $\sigma^{\prime}{ }_{3}$ through Eq. (6), the empirical 
models for axial strain, volumetric strain, and resilient modulus can be developed as represented by Eqs. (3), (4), and (5).

276

The 3D-plot of the empirical model prediction (i.e. Eqs. 3-5) and test results of axial strain, volumetric strain, and resilient modulus at different effective confining pressures and $N=10000$ are shown in Fig. 8 (a-c). Among all these test results, those of waste mixtures having $40 \% \mathrm{RC}$ was used only (independent set of data) for validating the model. It can be observed that the predicted results agree well with the measured data.

\section{Damping ratio and shear modulus}

The shear modulus $\mathrm{G}$ and the damping ratio $\mathrm{D}$ are the two key parameters needed to estimate the stiffness and energy absorbing capacity of soil. Damping is the loss of energy within a vibrating or a cyclically loaded system which is usually dissipated in the form of heat or breakage for granular materials; it is commonly used to measure the damping capacity for energy dissipation during dynamic or cyclic loading. The definition of the shear modulus and damping ratio is presented in the top right corner of Fig. 9 (e); where the area of the hysteretic loop $A_{2}$ in the shear stress-shear strain plain represents the energy dissipated during a loading cycle, while four times the area of the triangle $A_{1}$ is the maximum elastic energy absorbed during the cycle (Kokusho, 1980).

Fig. 9 (a-d) shows the shear modulus and damping ratio of SFS+CW+RC mixtures (SFS:CW=7:3) varying with loading cycles. It can be seen that at $\sigma_{3}^{\prime}=70 \mathrm{kPa}$ the shear modulus of the waste mixtures with $\geq 10 \%$ RC stays stable during the first 10 cycles and rises at a reducing rate after 10 cycles showing that the stiffness of the waste mixtures increases with the contraction of the specimens, while for the waste mixtures without rubber the shear modulus fluctuates marginally after 10 cycles indicating a stable stiffness of the 
traditional subballast (well-graded sand with gravel) tested by Suiker et al. (2005) is also shown in Fig. 9 (a). The tests conditions were the same with this study except the deviator stresses applied were different. Here only the tests result of $q=175$ and $91 \mathrm{kPa}$ are presented. It can be seen that the shear modulus increases as the deviator stress increases, and thus it can be estimated that when $q=112 \mathrm{kPa}$ at $\sigma^{\prime}{ }_{3}=70 \mathrm{kPa}$ (same with this study), the value of the shear modulus of subballast would be similar with $\mathrm{SFS}+\mathrm{CW}+\mathrm{RC}$ mixtures having $0 \% \mathrm{RC}$. Therefore, only the waste mixtures with $R C \leq 10 \%$ have acceptable stiffness comparing with traditional subballast.

Unlike the shear modulus, the damping ratio of the waste mixtures having $\mathrm{RC} \geq 10 \%$ decreases with the loading cycles at a decreasing rate and tends to achieve a similar value after 10000 cycles (Fig. 9c and d). This is because the inclusion of rubber crumbs increases the area of the hysteretic loop, but as RC contents increase the hysteretic loop becomes more inclined, which then causes a rapid increase in the area of the triangle $A_{1}$ (e.g. Fig. 2c), and this also suggests that the damping capacity of the waste mixtures with $\geq 10 \% \mathrm{RC}$ is similar at high loading cycles, while for the waste mixtures without rubber the value of the damping ratio is stable albeit a little fluctuation after 10 cycles. As with previous studies of rubbersand mixtures (e.g. Li et al., 2016; Zheng \& Kevin, 2000), the shear modulus decreases with increasing RC contents because of the low stiffness of rubber materials (Fig. 9a), and as the effective confining pressure increases the shear modulus increases (Fig. 9b). However, the damping ratio increases with the inclusion of $\mathrm{RC}$ contents but decreases with the effective confining pressure, which is opposite to the shear modulus (Fig. 9c and d).

Fig. 9 (e and f) shows the evolution of the shear modulus and damping ratio with $\mathrm{RC}$ contents and different effective confining pressures at 10000 cycles as the value of shear modulus and damping ratio becomes stable after 10000 cycles (e.g. Figs. 9 a-d). It can be noted that the 
behavior of shear modulus and the damping ratio is governed by the $\mathrm{RC}$ content inside the SFS+CW+RC mixtures. When $R C<20 \%$, the shear modulus decreases and the damping ratio increases as $\mathrm{RC}$ contents increase, while when $R C>20 \%$ both the shear modulus and the damping ratio change a little indicating that rubber crumbs have formed the skeleton of the whole specimen and the specimens behave rubber-like. It is worthy to note that when $10 \% \leq R C \leq 20 \%$, only a minor increase happens to the damping ratio with the addition of $\mathrm{RC}$, thus $10 \% \mathrm{RC}$ is sufficient for the purpose of energy absorbing.

Fig. 10 ( $\mathrm{a}$ and $\mathrm{b}$ ) compares the shear modulus and damping ratio of $\mathrm{SFS}+\mathrm{CW}+\mathrm{RC}$ mixtures with traditional subballast (after Suiker et al., 2005). It is evident from these plots that RC has a significant influence on the shear modulus $(\mathrm{G})$ of SFS+CW mixtures and not surprisingly, $\mathrm{G}$ decreases with the increase in rubber contents, $\mathrm{RC} \%$. Moreover, $\mathrm{G}$ decreases with an increase in the shear strain irrespective of the magnitude of RC. The variation of $\mathrm{G}$ with shear strain for SFS+CW+RC compares well with the subballast material reported by Suiker et al. (2005). Fig. 10 (b) also shows the effect of RC on the damping ratio of SFS+CW mixtures. It is clear from Fig. 10 (b) that damping ratio increases with both $\mathrm{RC}$ and shear strain. Two to three fold increase in damping ratio was observed for the SFS+CW blends with an addition of $10 \% \mathrm{RC}$.

It is important to note that the addition of just $10 \% \mathrm{RC}$ to the SFS+CW mixture has enabled to produce a sufficient shear modulus, typically required for a subballast material, besides showing a higher damping property than the mixtures without RC. These results further strengthen the prospect of this mixture as a suitable alternative material for the subballast layer in rail tracks.

\section{Energy absorbing analysis}


Fig. 11 illustrates how the total input energy is consumed by the energy absorbing layer (EAL). Generally, the total input energy cannot be totally absorbed by the energy absorbing layer (EAL), so part of the total energy will be transferred to the substructure, while the energy absorbed by the EAL will be dissipated by heat and breakage or turned into permanent deformation $\left(\varepsilon_{1}\right.$ and $\left.\varepsilon_{v}\right)$. The dissipated energy represented by the area of the hysteresis loop is due to: (a) the recoverable deformation of particles, (b) the friction of particles, and (c) particle breakage (Li et al., 2016; Zheng \& Kevin, 2000). The recoverable deformation is reflected by the resilient modulus $M_{R}$ (e.g. Eq. 2). In the SFS+CW+RC mixtures, SFS and CW particles barely deform whereas the rubber crumbs deform heavily and particle deformation can be partially recovered during unloading. The friction of particles is evaluated by the peak friction angle $\phi_{\text {peak }}^{\prime}$ obtained from drained monotonic triaxial tests (to focus on the cyclic loading results, the monotonic tests are not included in this paper). Particle breakage mainly occurs to $\mathrm{CW}$ particles, and it is evaluated by the breakage index (BI) which is proposed by Indraratna et al. (2005).

In Fig. 11 the cyclic loading test results are also presented to better illustrate the influence of the RC content on the energy consumption distribution. For brevity, only the typical case of ${\sigma^{\prime}}_{3}=70 \mathrm{kPa}$ at $N=10000$ is described herein, as the results obtained for other confining pressure levels are similar and the dynamic properties (e.g. strains, resilient modulus, shear modulus, and damping ratio) of the other SFS $+\mathrm{CW}+\mathrm{RC}$ mixtures also seem to stabilize when approaching 10000 cycles. It is found that when the RC content in the SFS+CW+RC mixtures increases, the energy absorbed by the EAL increases as the area of the shear stressshear strain triangle increases (Fig. 11a), indicating that less energy will be transmitted to substructure, but accordingly, the energy results in permanent strains (Fig. 11b) and the dissipated energy increases (Fig. 11c). The rise in the dissipated energy mainly results from 
the increasing recoverable deformation $\left(M_{R}\right.$ decreases, Fig. 11d) as the shear strength (Fig. 11e) and particle breakage (Fig. 11f) decrease with the inclusion of RC.

Therefore, it can be seen that more rubber ensures the EAL (SFS+CW+RC mixtures) to have a higher energy absorbing capacity, less particle breakage, but adverse effects such as higher deformation, a lower resilient modulus, and less shear strength are also generated. Note also that $10 \%$ of RC already has a promising energy absorbing capacity, damping ratio, and less particle breakage than the mixtures without rubber, while tolerating an acceptable reduction in shear modulus and a bit increase in strains, therefore $10 \% \mathrm{RC}$ is the recommended amount for the energy absorbing layer.

\section{Conclusions}

In this study stress-controlled cyclic triaxial tests were conducted on $\mathrm{SFS}+\mathrm{CW}+\mathrm{RC}$ mixtures with SFS:CW=7:3, while the RC contents changed from $0 \%$ to $40 \%$ to investigate how the amount of rubber would affect the permanent deformation, resilient deformation, damping ratio, and shear modulus under cyclic loading conditions. The energy absorbing capacity of these waste mixtures was also analysed based on the comprehensive test results. The following significant findings were obtained.

- The permanent axial strain and volumetric strain increased with the increasing RC contents and confining pressures. A 3D empirical model of strains related to the RC content and confining pressure was developed, and showed a good agreement with test results.

- The resilient deformation increased with the inclusion of rubber crumbs, but it decreased as the confining pressure increased reflected by an increase of the resilient modulus. A 3D empirical relationship was also found for the resilient modulus with RC contents and confining pressures, and the model matched the test results very well. 
- The addition of RC also caused the shear modulus to decrease and the damping ratio to increase; this indicated that the stiffness of the waste mixtures decreased, while the absorbed energy dissipated to heat or breakage became more efficient. It was also found that the behavior of shear modulus and damping ratio was controlled by the

\section{Acknowledgements}

410

The first author would like to acknowledge the financial assistance provided by the China

Scholarship Council. The assistance provided by industry (ASMS and South 32) in relation to the procurement of material used in this study is gratefully acknowledged. The assistance in the laboratory from Mr. Richard Berndt and the occasional technical feedback from A/Prof

\section{References}



test method for load controlled cyclic triaxial strength of soil, ASTM International, West Conshohocken, PA, USA.

ASTM D6270, American Society for Tests and Materials 2008 (R2012), Standard practice 420 for use of scrap tyres in civil engineering applications, ASTM D International, West Conshohocken, PA, USA.

422 Bian, XC, Jiang, JQ, Sun, DD, Li, W \& Li, XM 2016, 'Cyclic and postcyclic triaxial testing 423 of ballast and subballast', J. Mater. Civ. Eng., vol. 28, No. 7, pp. 04016032(1)- (12).

Blunden, B \& Gray, A 2006. Coal Wash data and Product Specification: fill. BHP BillitonIllawarra Coal, Processing and Logistics, Wollongong, NSW, Australia, 31.

Chen, QS \& Indraratna, B 2015, 'Deformation behavior of lignosulfonate-treated sandy silt 427 under cyclic loading', J. Geotech. Geoenviron. Eng., vol. 141, No. 1, pp. 06014015-1-5.

Chiaro, G, Indraratna, B, Tasalloti, SMA \& Rujikiatkamjorn, C 2015, 'Optimisation of coal 429 wash-slag blend as a structural fill', Ground Improvement, vol. 168, No. GI1, pp. 33-44.

Heitor, A, Indraratna, B, Kaliboullah, CI, Rujikiatkamjorn, C \& McIntosh, G 2016, 'Drained 431 and undrained shearing behavior of compacted coal wash', J. Geotech. Geoenviron. Eng., vol. 142, No. 5, pp. 04016006-1-10.

Indraratna, B, Gasson, I \& Chowdhury, RN 1994, 'Utilization of compacted coal tailings as a 434 structural fill', Can. Geotech. J., vol. 31, No. 5, pp. 614-623.

Indraratna B, Lackenby, J \& Christie, D 2005, 'Effect of confining pressure on the degradation of ballast under cyclic loading', Géotechnique, vol. 55, No. 4, pp. 325-328. 
Indraratna, B, Raut, AK \& Khabbaz, H 2007, 'Constriction-based retention criterion for granular filter design', J. Geotech. Geoenviron. Eng., vol. 133, No. 3, pp. 266-276.

Indraratna, B, Salim, W \& Rujihiatkamjorn, C 2011, Advanced rail geotechnology-ballasted track, CRC Press/Balkema, The Netherlands.

Indraratna, B, Biabani, MM \& Nimbalkar, S 2014, 'Behavior of Geocell-reinforced subballast subjected to cyclic loading in plane-strain condition', J. Geotech. Geoenviron. Eng., vol. 141, No. 1, pp. 04014081(1)- (16).

Juan, LM, Claisse, P \& Ganjian, E 2011, 'Effect of steel slag and Portland cement in the rate of hydration and strength of blast furnace slag pastes', J. Mater. Civ. Eng., vol. 23, No. 2, pp.153-160.

Kim, HK \& Santamarina, JC 2008, 'Sand-rubber mixtures (large rubber chips)', Can. Geotech. J., vol. 45, pp. 1457-1466.

Kokusho, T 1980, 'Cyclic triaxial test of dynamic soil properties for wide strain range', Soil and Foundations, vol. 20, No. 2, pp. 45-60.

Lackenby, J, Indraratna, B, Mcdowell, G \& Christie, D 2007, 'Effect of confining pressure on ballast degradation and deformation under cyclic triaxial loading', Géotechnique, vol. 57, No. 6, pp. 527-536.

Lee, JH, Salgado, R, Bernal, A \& Lovell, CW 1999, 'Shredded tires and rubber-sand as lightweight backfill', J. Geotech. Geoenviron. Eng., vol. 125, No. 2, pp. 132-141.

Lee, J, Dodds, J \& Santamarina, JC 2007, 'Characteristics of rubber-sand particle mixtures according to size ratio', J. Mater. Civ. Eng., vol. 19, No. 2, pp. 179-184.

Leo, M \& Mladen, V 2003, 'Effect on soil secant shear modulus at small cyclic strains', $J$. Geotech. Geoenviron. Eng., vol. 129, No. 6, pp. 536-549. 
Li, B, Huang, MS \& Zeng, XW 2016, 'Dynamic behavior and liquefaction analysis of recycled-rubber sand mixtures', J. Mater. Civ. Eng., vol. 28, No. 11, pp. 04016122.

Lim, T \& Chu, J 2006, 'Assessment of use of spent copper slag for land reclamation', Waste Management Research, vol. 24, pp. 67-73.

Malasavage, N, Jagupilla, S, Grubb, D, Wazne, M, \& Coon, W 2012, 'Geotechnical

Performance of Dredged Material-Steel Slag Fines Blends: Laboratory and Field

Marachi, ND, Chan, CK \& Seed, HB 1972, 'Evaluation of properties of rockfill materials', J.

Soil Mech. Found. Div. ASCE, vol. 98, No. 1, pp. 95-114.

Mccallum, DA 2005, 'The hydrochemistry of steel and iron blast furnace slags. Part1:

470 Characterisation'. CSIRO Minerals.

Nazzal, MD \& Mohammad, LN 2010, 'Estimation of resilient modulus of subgrade soils for design of pavement structures', J. Mater. Civ. Eng., vol. 22, No. 7, pp. 726- 734.

NSW Environment Protection Authority (EPA), 2014. Resource Recovery Exemption, the

474 Protection of the Environment Operations (Waste) Regulation 2014 - The coal washery rejects exemption (http://www.epa.nsw.gov.au/resources/waste/rre14-coal-wash-rejects.pdf)

NSW Environment Protection Authority (EPA), 2014. Resource Recovery Exemption the

477 Protection of the Environment Operations (Waste) Regulation 2014 - The steel furnace slag exemption (http://www.epa.nsw.gov.au/resources/waste/rrel4-steel-furnace-slag.pdf)

479 Selig, ET \& Waters, JM 1994, Track technology and substructure management, Thomas Telford, London. 
Senetakis, K, Anastasiadis, A \& Pitilakis, K 2012, 'Dynamic properties of dry sand/rubber (SRM) and gravel/rubber (GRM) mixtures in a wide range of shearing strain amplitudes', $J$. Soil Dyn. Earthquake Eng., vol. 33, No. 1, pp. 38-53.

Shahu, JT, Kameswara Rao, NSV \& Yudhbir 1999, 'Parametric study of resilient response of tracks with a sub-ballast layer', Can. Geotech. J., vol. 36, No. 6, pp. 1137-1150.

Sheikh, MN, Mashiri, M, Vinod, J \& Tsang, H 2013, 'Shear and Compressibility Behavior of Sand-Tyre Crumb Mixtures', J. Mater. Civ. Eng., vol. 25, pp. 1366-1374.

Suiker, ASJ, Selig, ET \& Frenkel R 2005, 'Static and cyclic triaxial testing of ballast and subballast', J. Geotech. Geoenviron. Eng., vol. 131, No. 6, pp. 771-782.

Tasalloti, SMA, Indraratna, B, Rujikiatkamjorn, C, Heitor, A \& Chiaro, G 2015, 'A laboratory Study on the Shear Behavior of Mixtures of Coal Wash and Steel Furnace Slag as Potential Structural Fill', Geotechnical testing journal, vol. 38, No. 4, pp. 361-372.

Teixeira, PF, López-Pita, A, Casas-Esplugas, C, Bachiller, A \& Robusté, F 2006, 'Improvements in high-speed ballasted track design: Benefits of bituminous subballast layers', Transportation Research Record 1943, pp. 43-49.

Tsang, HH, Lo, SH, Xu, X \& Sheikh, MN 2012, 'Seismic isolation for low-to-medium-rise buildings using granulated rubber-soil mixtures: numerical study', Earthquake Engineering and Structural Dynamics, vol. 41, No. 14, pp. 2009-2024.

Xue, Y, Wu, S, Hou, H \& Zha, J 2006, 'Experimental investigation of basic oxygen furnace slag used as aggregate in asphalt mixture', J. Hazard. Mater., vol. 138, No. 2, pp. 261-268.

Yildirim, IZ \& Prezzi, M 2015, 'Geotechnical properties of fresh and aged basic oxygen furnace steel slag', J. Mater. Civ. Eng., vol. 27, No. 12, pp. 04015046-1-11. 
503 Zheng, YF \& Kevin, GS 2000, 'Dynamic properties of granulated rubber/sand mixtures', 504 Geotechnical Testing Journal, vol. 23, No. 3, pp. 338-344. 
506 Fig. 1 Particle size distribution of the waste materials and the target PSD

507 Fig. 2 Hysteretic cycles of waste mixtures (SFS:CW=7:3) tested at $\sigma^{\prime}{ }_{3}=70 \mathrm{kPa}$ :(a) having $50810 \% \mathrm{RC}$ at different cycles; (b) having 30\% RC at different cycles; (c) having different RC 509 contents at 10000 cycles

510 Fig. 3 Permanent strains under cyclic loading for SFS+CW+RC mixtures (SFS:CW=7:3)

511 having different $\mathrm{RC}$ contents tested with $\mathrm{CSR}=0.8$ and $f=5 \mathrm{~Hz}$ : (a-c) axial strain, (d-f) 512 volumetric strain

513 Fig. 4 Influence of RC content on Dilation Behaviour of Waste Mixtures

514 Fig. 5 Resilient modulus of SFS+CW+RC mixtures (SFS:CW=7:3) having different RC 515 contents tested with $\mathrm{CSR}=0.8$ and $f=5 \mathrm{~Hz}$ and at: (a) ${\sigma^{\prime}}_{3}=10 \mathrm{kPa}$; (b) $\sigma_{3}^{\prime}=40 \mathrm{kPa}$; $516 \quad$ (c) $\sigma_{3}^{\prime}=70 \mathrm{kPa}$

517 Fig. 6 Radial strain of SFS+CW+RC mixtures (SFS:CW=7:3) having different RC contents at: (a) $\mathrm{N}=10$ cycles; (b) $\mathrm{N}=10000$ cycles

519 Fig. 7 Cyclic loading test results of SFS+CW+RC mixtures with SFS:CW=7:3 and different 520 RC contents at 10000 cycles: (a) axial strain; (b) volumetric strain; (c) resilient modulus

521 Fig. 8 Relationship between $\sigma_{3}^{\prime}, R_{b}$, and (a) $\varepsilon_{1}$; (b) $\varepsilon_{v}$; (c) $M_{R}$, and surface represent model 522 predictions (Eq. 3, Eq. 4, and Eq. 5) at $\mathrm{N}=10000$

523 Fig. 9 Shear modulus and damping ratio under cyclic loading: (a, b) shear modulus of waste 524 mixtures (SFS:CW=7:3) changing with loading cycles; (c, d) damping ratio of waste mixtures 525 (SFS:CW=7:3) changing with loading cycles; (e, f) shear modulus and damping ratio of waste mixtures (SFS:CW=7:3) changing with RC contents at 10000 cycles 
527 Fig. 10 (a) Shear modulus of SFS+CW+RC mixtures changing with shear strain amplitude 528 and comparison with traditional subballast (after Suiker et al., 2005); (b) Damping ratio of 529 SFS+CW+RC mixtures changing with shear strain amplitude

530 Fig. 11 Energy consumption flow chart and comprehensive properties of waste mixtures 531 (SFS:CW=7:3) having different RC contents, at $\sigma_{3}^{\prime}=70 \mathrm{kPa}$ and $\mathrm{N}=10000$ 


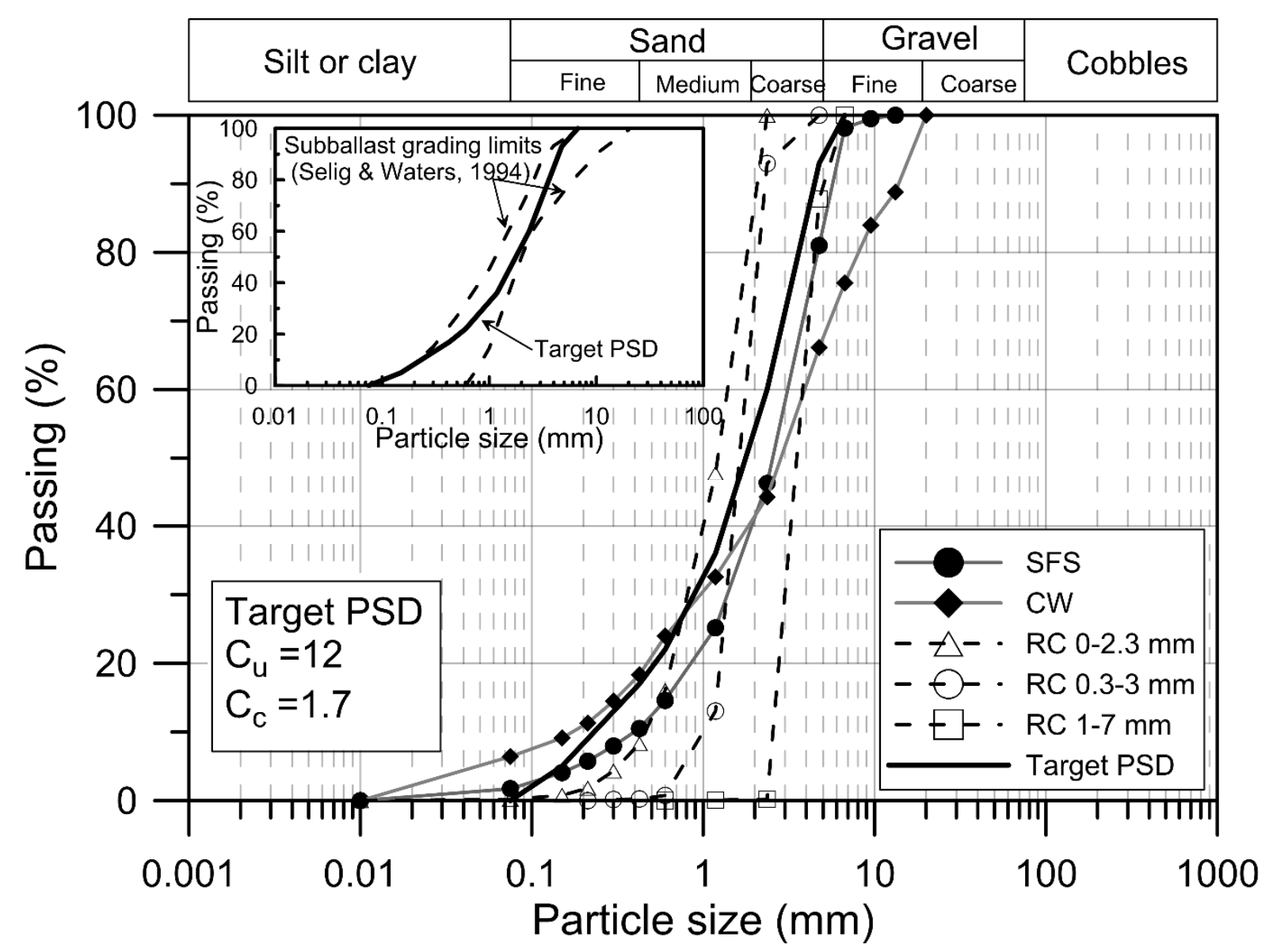

Fig. 1 Particle size distribution of the waste materials and the target PSD 

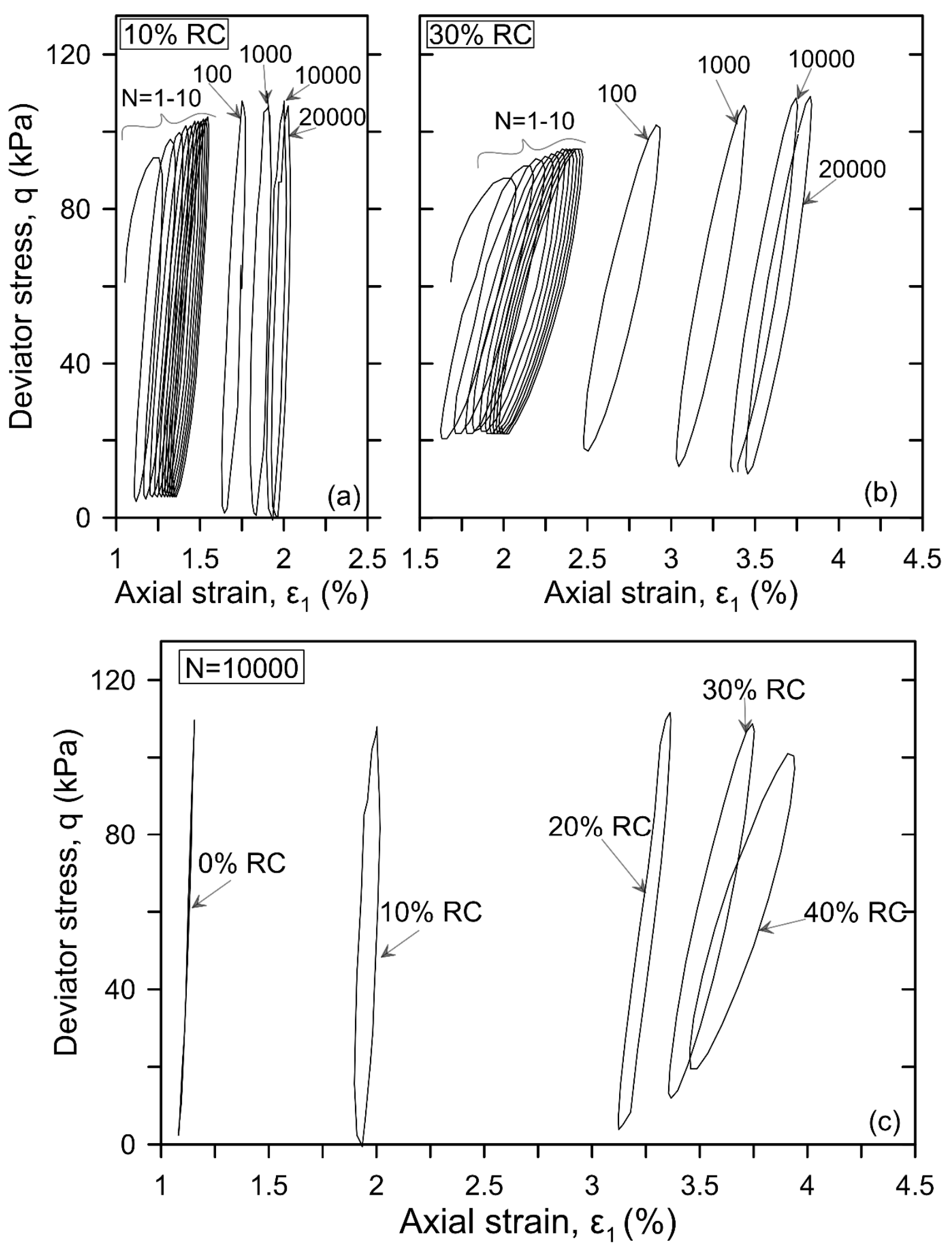

535 Fig. 2 Hysteretic cycles of waste mixtures (SFS:CW=7:3) tested at ${\sigma^{\prime}}_{3}=70 \mathrm{kPa}$ :(a) having $53610 \% \mathrm{RC}$ at different cycles; (b) having 30\% RC at different cycles; (c) having different RC contents at 10000 cycles 

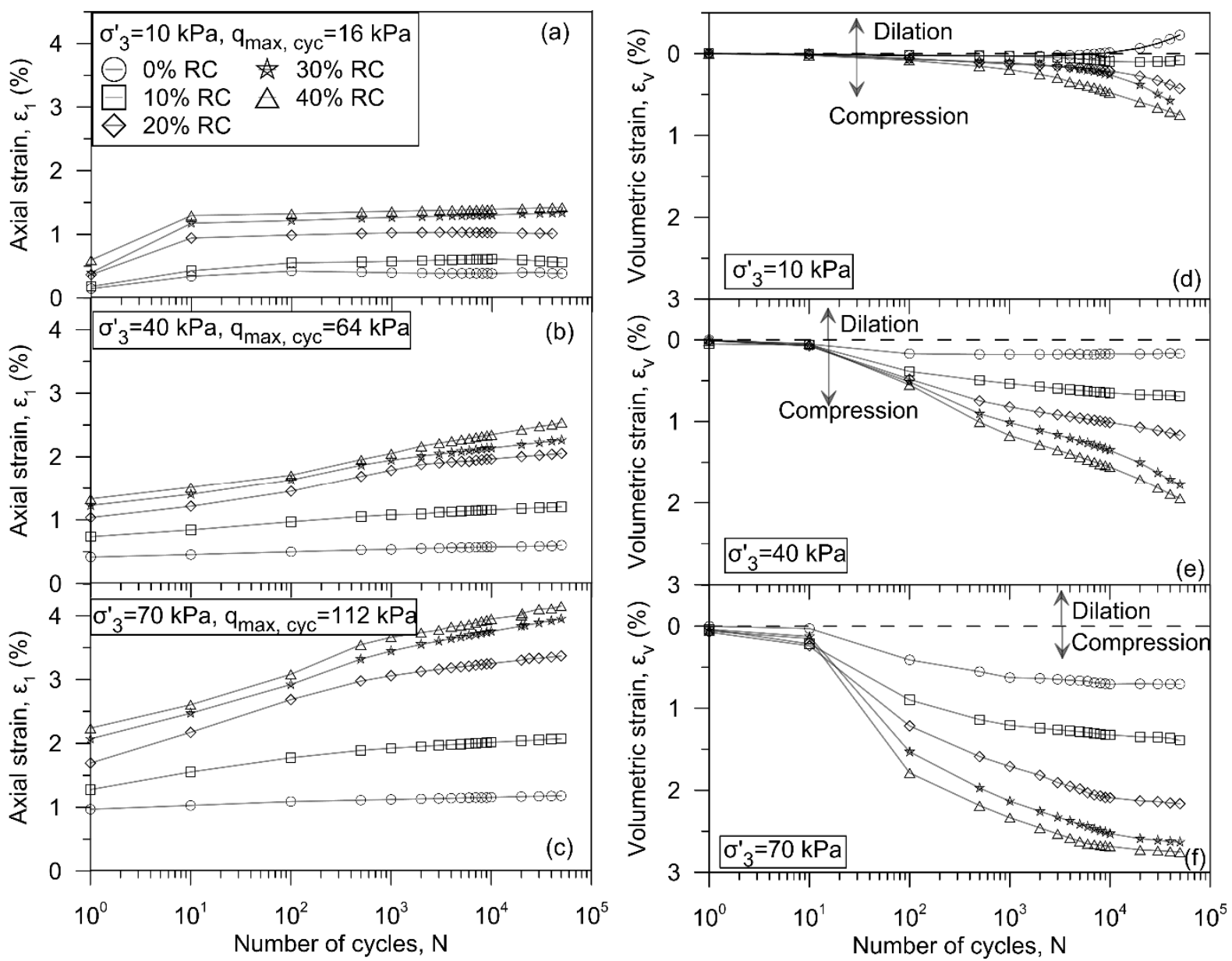

Fig. 3 Permanent strains under cyclic loading for SFS+CW+RC mixtures (SFS:CW=7:3)

540 having different $\mathrm{RC}$ contents tested with $\mathrm{CSR}=0.8$ and $f=5 \mathrm{~Hz}$ : (a-c) axial strain, (d-f) 


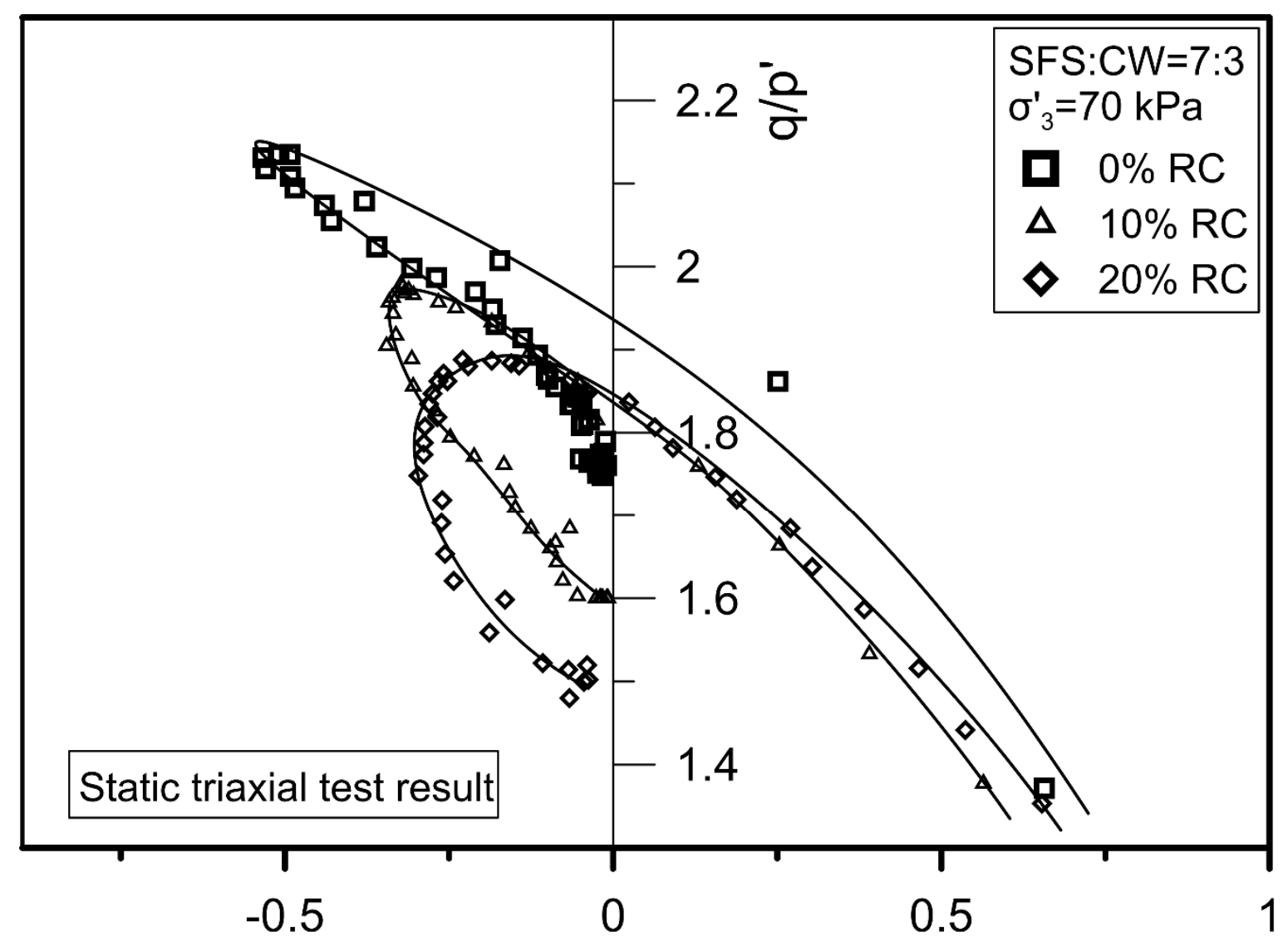

Dilatancy, $\mathrm{d} \varepsilon_{\mathrm{v}}^{\mathrm{p}} / \mathrm{d} \varepsilon_{\mathrm{q}}^{\mathrm{p}}$ 


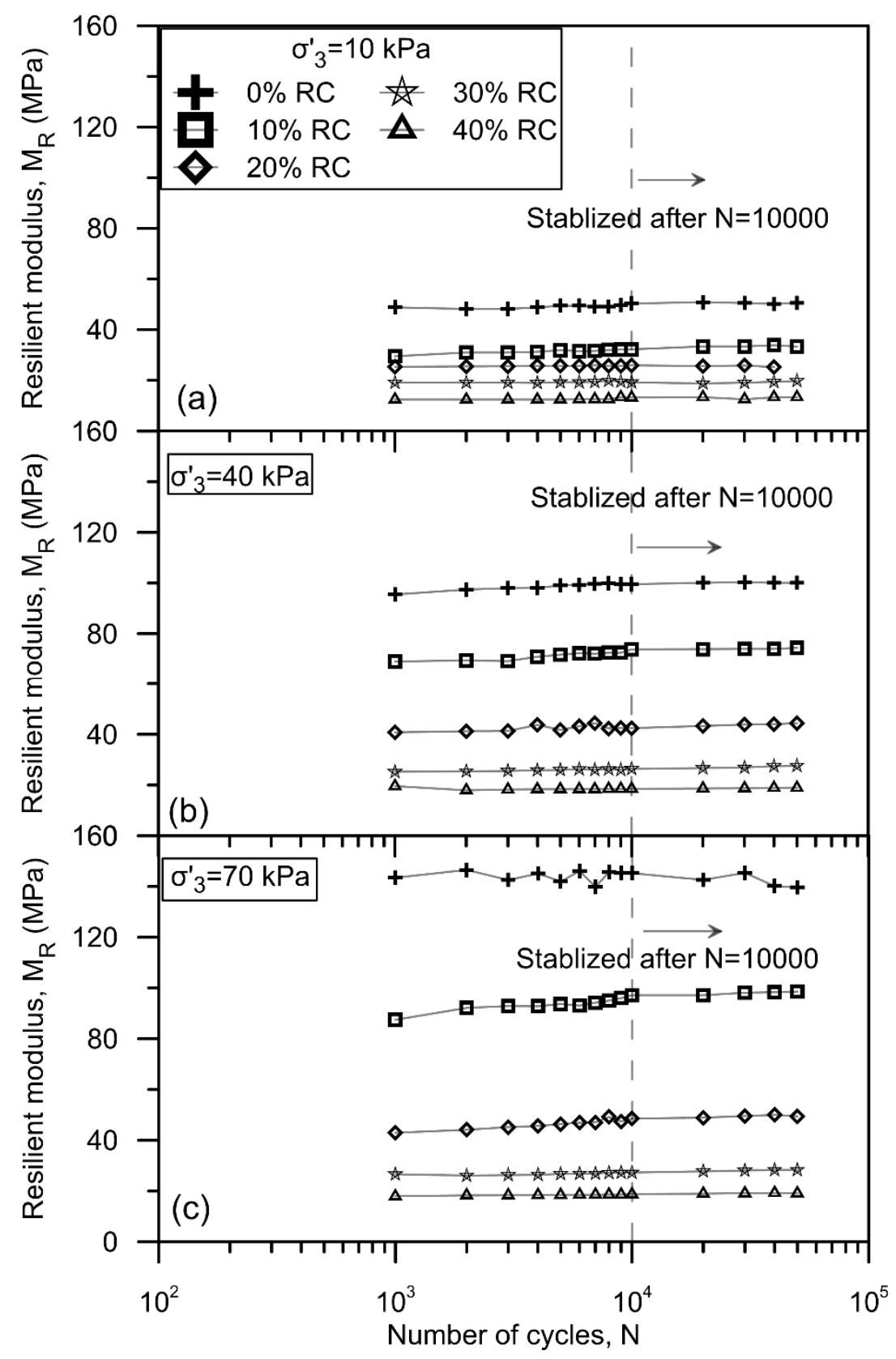

547 Fig. 5 Resilient modulus of SFS+CW+RC mixtures (SFS:CW=7:3) having different RC

548 contents tested with $\mathrm{CSR}=0.8$ and $f=5 \mathrm{~Hz}$ and at: (a) $\sigma_{3}^{\prime}=10 \mathrm{kPa}$; (b) $\sigma_{3}^{\prime}=40 \mathrm{kPa}$;

(c) $\sigma_{3}^{\prime}=70 \mathrm{kPa}$ 


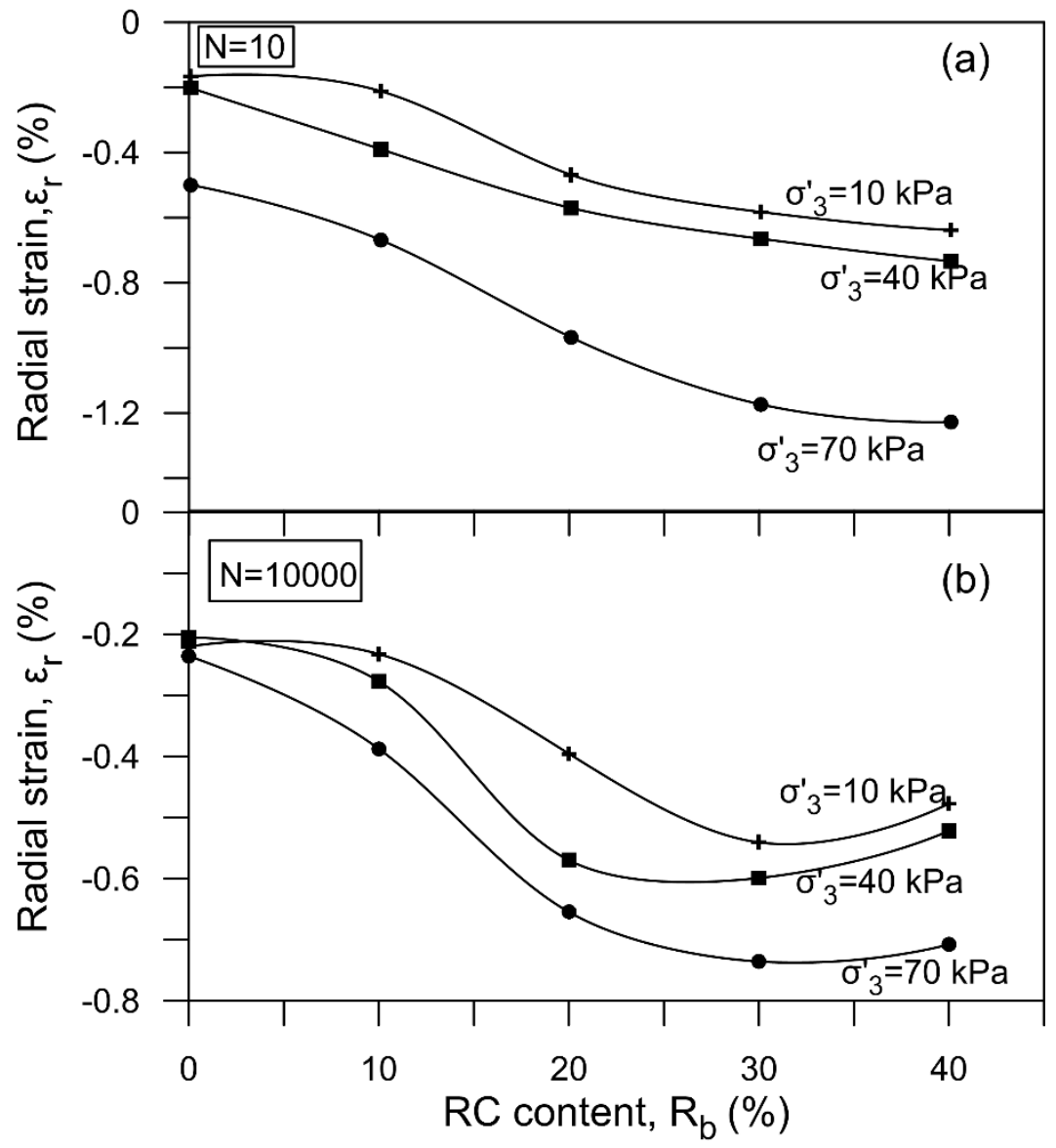

551

552 Fig. 6 Radial strain of SFS+CW+RC mixtures (SFS:CW=7:3) having different RC contents

at: (a) $\mathrm{N}=10$ cycles; (b) $\mathrm{N}=10000$ cycles 


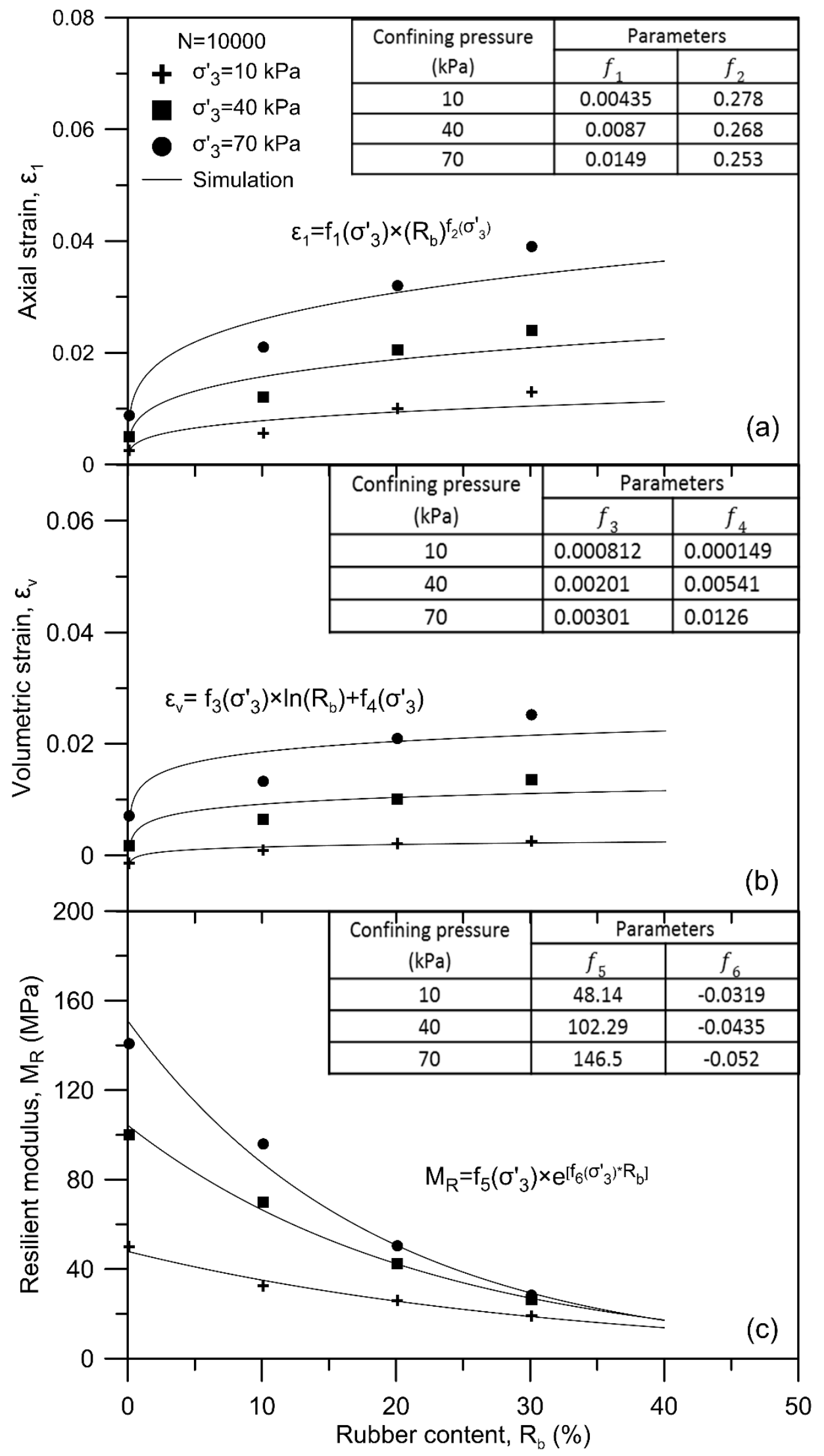

Fig. 7 Cyclic loading test results of SFS+CW+RC mixtures with SFS:CW=7:3 and different 

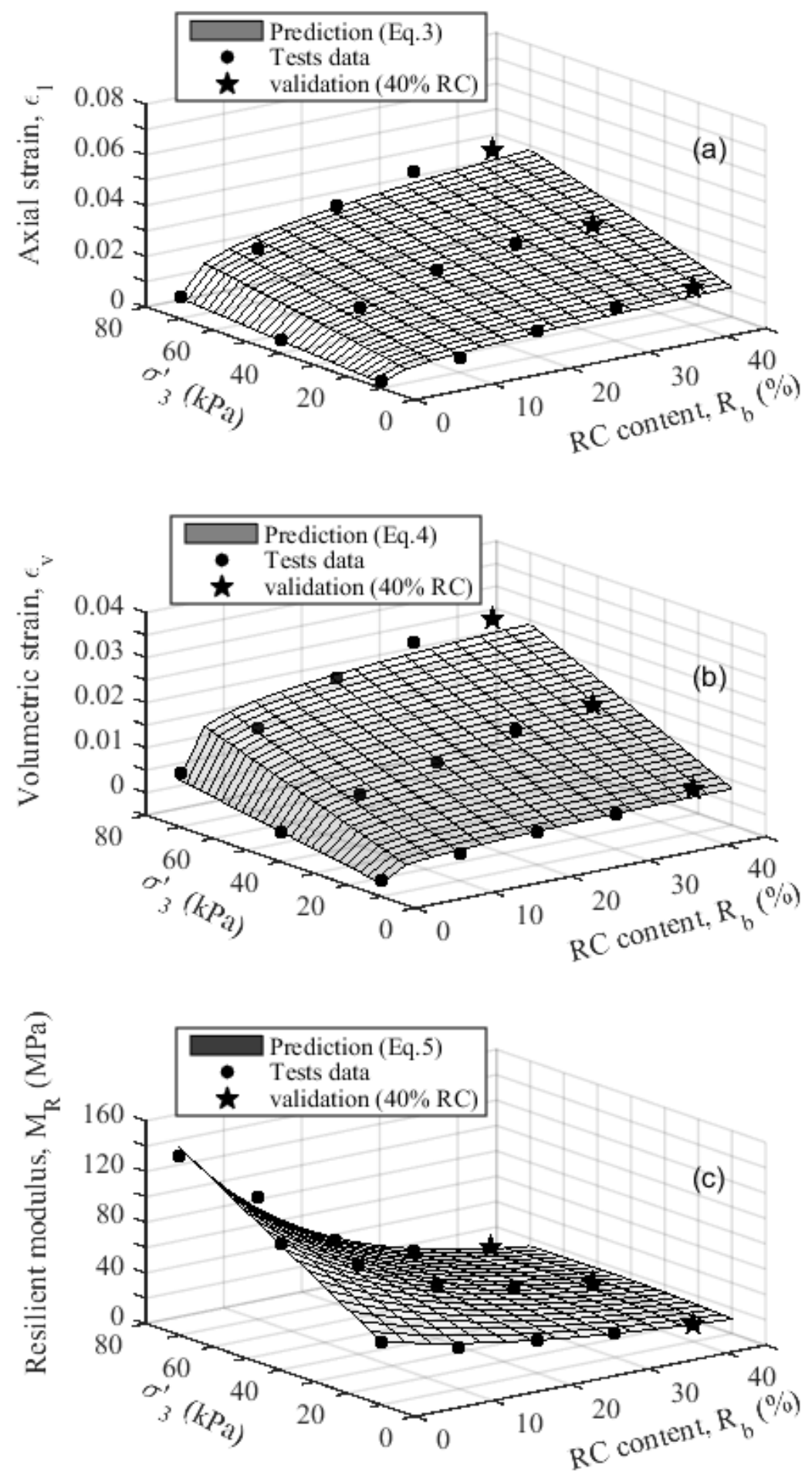

558 Fig. 8 Relationship between $\sigma_{3}^{\prime}, R_{b}$, and (a) $\varepsilon_{1}$; (b) $\varepsilon_{v}$; (c) $M_{R}$, and surface represent model predictions (Eq. 3, Eq. 4, and Eq. 5) at $\mathrm{N}=10000$ 

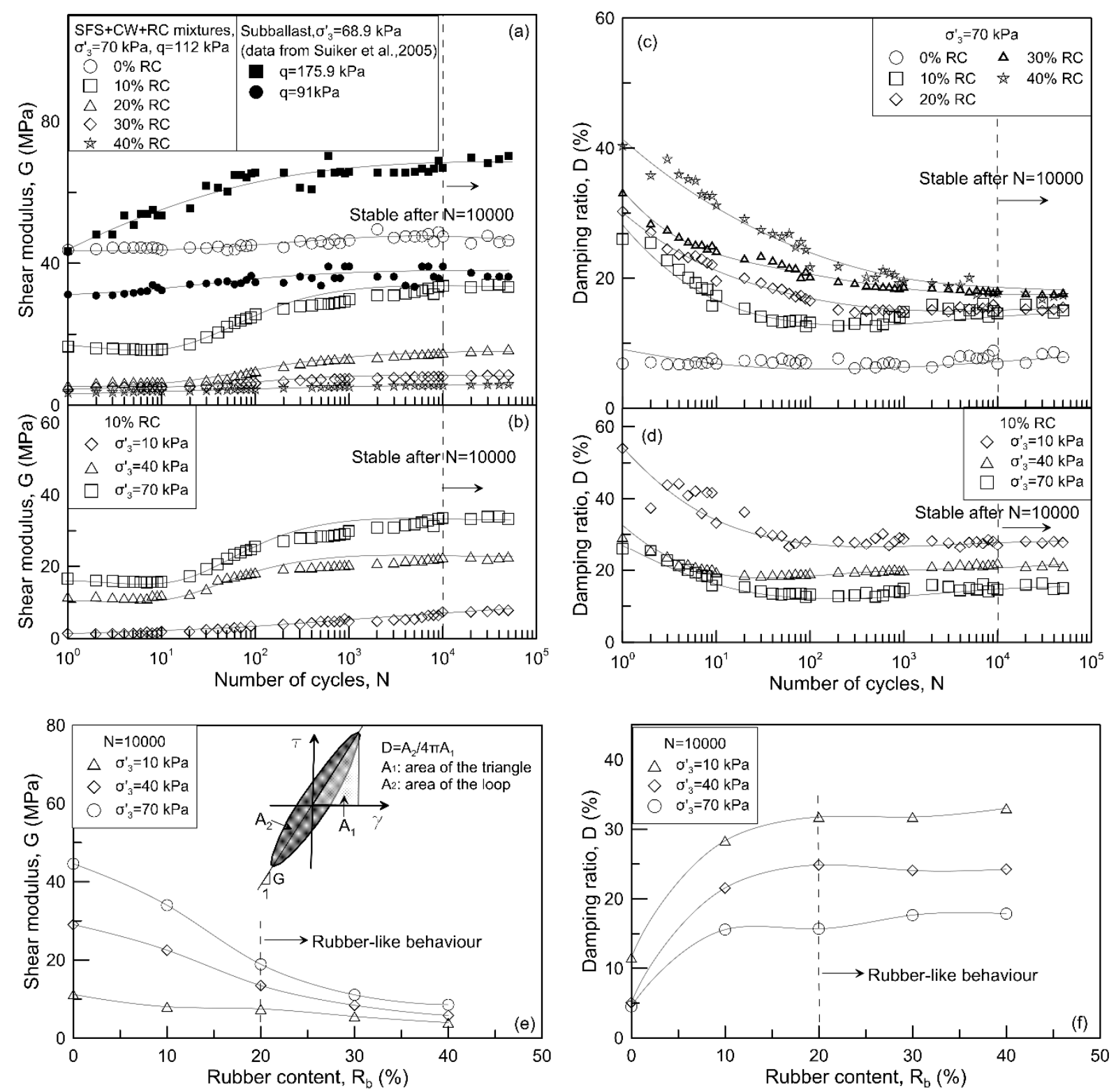

561 Fig. 9 Shear modulus and damping ratio under cyclic loading: (a, b) shear modulus of waste mixtures (SFS:CW=7:3) changing with loading cycles; (c, d) damping ratio of waste mixtures (SFS:CW=7:3) changing with loading cycles; $(\mathrm{e}, \mathrm{f})$ shear modulus and damping ratio of waste mixtures (SFS:CW=7:3) changing with RC contents at 10000 cycles 


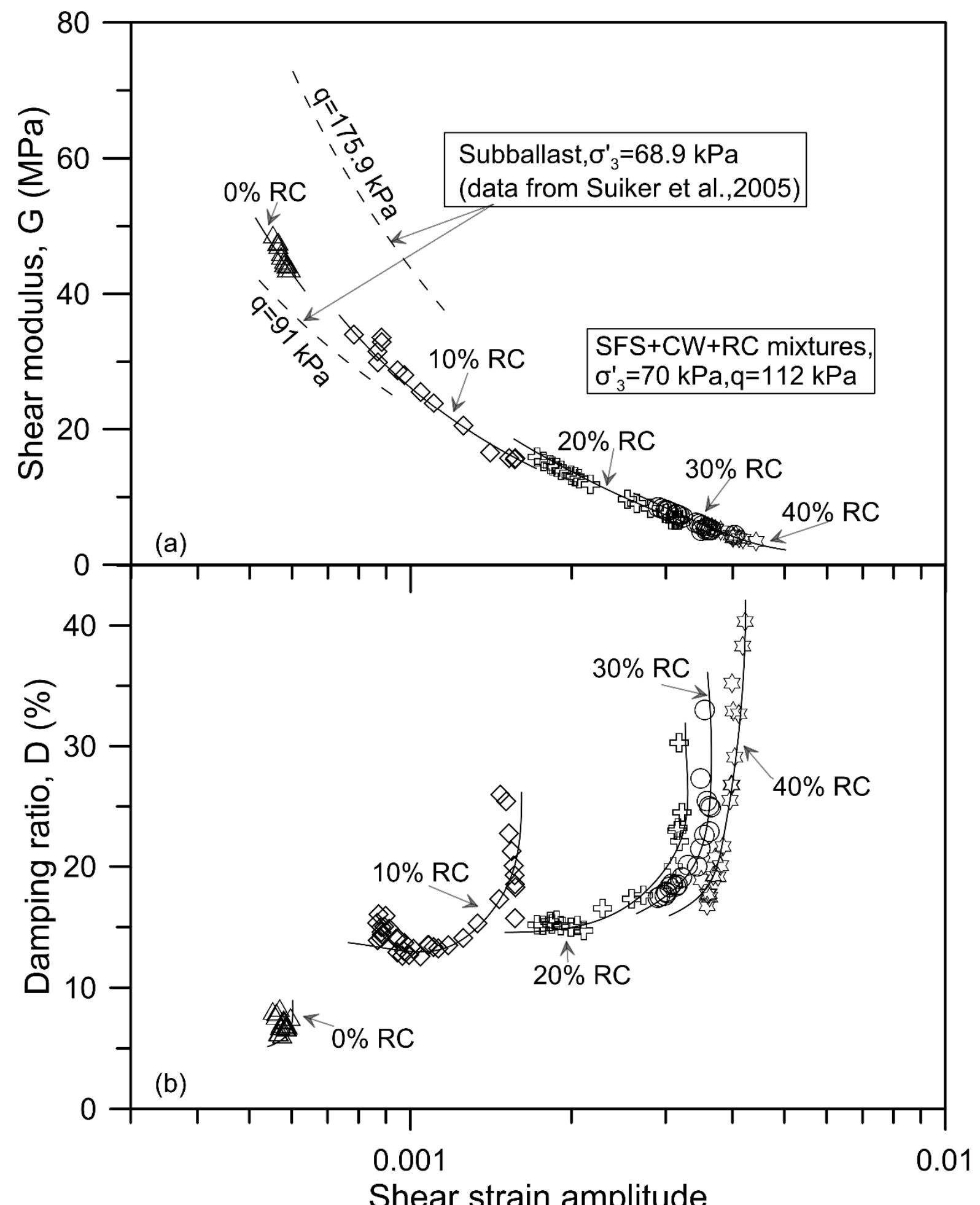

Fig. 10 (a) Shear modulus of SFS+CW+RC mixtures changing with shear strain amplitude and comparison with traditional subballast (after Suiker et al., 2005); (b) Damping ratio of 


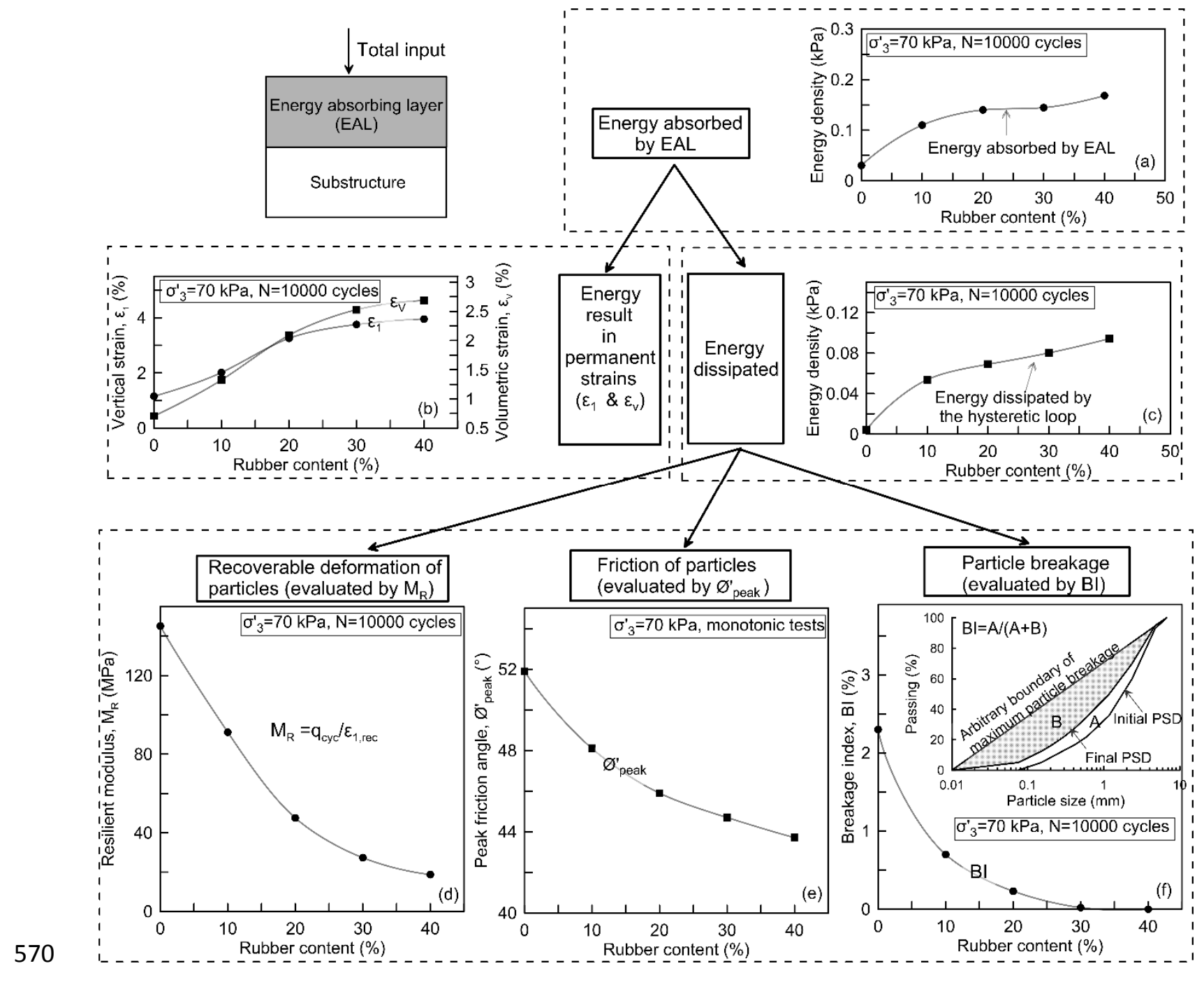

571 Fig. 11 Energy consumption flow chart and comprehensive properties of waste mixtures

572 (SFS:CW=7:3) having different RC contents, at $\sigma_{3}^{\prime}=70 \mathrm{kPa}$ and $\mathrm{N}=10000$ 
575 Table 1 Typical chemical composition of CW (Blunden \& Gray, 2006);

576 Table 2 Typical chemical composition of SFS (McCallum, 2005);

577 Table 3 Basic geotechnical properties of SFS, CW, RC and their mixtures;

578 Table 4 Value of $\mathrm{k}_{n 1}$ and $\mathrm{k}_{n 2}$.

579

580

581

Table 1 Typical chemical composition of CW (Blunden \& Gray, 2006)

\begin{tabular}{cc}
\hline Component & Proportion $(\%)$ \\
\hline Ash & 65.6 \\
Carbon & 24.3 \\
Volatiles & 14.4 \\
Hydrogen & 1.9 \\
Nitrogen & 0.55 \\
Sulphur & 0.23 \\
Phosphor & 0.02 \\
\hline
\end{tabular}

582

Table 2 Typical chemical composition of SFS (McCallum, 2005)

\begin{tabular}{cc}
\hline Component & Proportion $(\%)$ \\
\hline $\mathrm{SiO} 2$ & 12.5 \\
$\mathrm{Al} 2 \mathrm{O} 3$ & 2.8 \\
$\mathrm{CaO}$ & 38.3 \\
$\mathrm{MgO}$ & 9.9 \\
$\mathrm{Fe} 2 \mathrm{O} 3$ & 30 \\
$\mathrm{MnO}$ & 3.7 \\
$\mathrm{TiO} 2$ & 1.2 \\
Others & 1.6 \\
\hline
\end{tabular}


Table 3 Basic geotechnical properties of SFS, CW, RC and their mixtures

\begin{tabular}{|c|c|c|c|c|c|c|}
\hline Material & SFS:CW & $\mathrm{RC}(\%)$ & $\boldsymbol{G}_{\boldsymbol{s}}$ & $\begin{array}{c}\gamma_{d \max } \\
\left(\boldsymbol{k N} / \boldsymbol{m}^{3}\right)\end{array}$ & $\begin{array}{c}\text { OMC } \\
(\%)\end{array}$ & $\begin{array}{r}\text { CBR } \\
(\%)\end{array}$ \\
\hline SFS & - & - & 3.43 & - & - & - \\
\hline $\mathrm{CW}$ & - & - & 2.11 & - & - & - \\
\hline $\mathrm{RC}$ & - & - & 1.15 & - & - & - \\
\hline SFS70+CW30 & \multirow{5}{*}{$7: 3$} & 0 & 2.89 & 20.30 & 11.5 & 58 \\
\hline SFS63+CW27+RC10 & & 10 & 2.51 & 17.57 & 12.5 & 43 \\
\hline SFS56+CW24+RC20 & & 20 & 2.22 & 15.50 & 13 & 15 \\
\hline SFS49+CW21+RC30 & & 30 & 1.99 & 13.83 & 14 & 6 \\
\hline SFS42+CW18+RC40 & & 40 & 1.80 & 12.40 & 15 & 4 \\
\hline
\end{tabular}

586 The mixtures are expressed as SFS+CW+RC, and the numbers after SFS, CW, and RC are

587 the percentages of steel furnace slag, coal wash, and rubber crumbs by weight.

588

589

590

591

Table 4 Value of $\mathrm{k}_{n 1}$ and $\mathrm{k}_{n 2}$

\begin{tabular}{ccc}
\hline $\begin{array}{c}\text { Parameter } \\
\text { No. }\end{array}$ & $\mathbf{k}_{\boldsymbol{n} \mathbf{1}}$ & $\mathbf{k}_{\boldsymbol{n} \mathbf{2}}$ \\
\hline $\boldsymbol{n}=\mathbf{1}$ & $1.7 \times 10^{-4}$ & $2.28 \times 10^{-3}$ \\
$\boldsymbol{n}=\mathbf{2}$ & $-4.17 \times 10^{-4}$ & 0.283 \\
$\boldsymbol{n}=\mathbf{3}$ & $3.7 \times 10^{-5}$ & $4.8 \times 10^{-4}$ \\
$\boldsymbol{n}=\mathbf{4}$ & $2.1 \times 10^{-4}$ & $2.2 \times 10^{-3}$ \\
$\boldsymbol{n}=\mathbf{5}$ & 1.639 & 33.4 \\
$\boldsymbol{n}=\mathbf{6}$ & $-3.35 \times 10^{-4}$ & -0.0291 \\
\hline
\end{tabular}

592 